\title{
ESTUDO DA DEGRADAÇÃO DE FACHADA DE EDIFÍCIO EM BRASÍLIA/DF - ELABORAÇÃO DE CURVA DOSE-RESPOSTA
}

\author{
BAUER, ELTON \\ Professor \\ Universidade de Brasília \\ Distrito Federal; Brasil \\ elbauerlem@gmail.com
}

\author{
CAVALCANTE, ANTONIO \\ Eng. civil, aluno de mestrado \\ Universidade de Brasília \\ Distrito Federal; Brasil \\ caio_andradecavalcante@hotmail.com
}

\author{
LUCENAS, RAYNNARA \\ Eng. civil, aluna de mestrado \\ Universidade de Brasília \\ Distrito Federal; Brasil \\ lucenasraynnara@gmail.com \\ NETO, EDUARDO \\ Eng. civil, aluno de mestrado \\ Universidade de Brasília \\ Distrito Federal; Brasil \\ eduardo.neto@ctec.ufal.br
}

\author{
PEREIRA, VICTOR \\ Eng. civil, aluno de mestrado \\ Universidade de Brasília \\ Distrito Federal; Brasil \\ victorpereira.eng@gmail.com
}

\section{RESUMO}

A influência dos agentes climáticos sobre as fachadas pode ocasionar degradação, sendo necessária a supervisão desta ação. Algumas técnicas vem sendo desenvolvidas e com este intuito, pesquisadores da Universidade de Brasília desenvolveram o Método de Mensuração da Degradação (MMD) que permite essa avaliação quantitativa da degradação, resultando em um índice denominado Fator Geral de Danos (FGD). O objetivo deste estudo é realizar uma conexão entre FGD e incidência solar para fachada em revestimento cerâmico de um edifício localizado em Brasília-DF. A quantificação dos agentes foi realizada por meio de simulação higrotérmica, aplicando o sofwtare WUFI. A influência da radiação foi determinada pelo cálculo diário da amplitude térmica ou Delta T. Foi determinado o Indice de Incidência de Temperatura (Iit), sendo definidas 4 faixas de Delta-T e determinadas suas frequências de ocorrência. A resposta final é uma curva Dose-Resposta, que apresenta uma tencência exponencial e auxilia na investigação de degradação e vida útil.

Palavras-chave: fachadas, degradação, simulação higrotérmica, dose-resposta.

\section{ABSTRACT}

The influence of climatic agents on the façades can cause degradation, being necessary the supervision of this action. Some techniques have been developed and to this end, researchers from the University of Brasilia have developed the Degradation Measurement Method (MMD) that allows this quantitative assessment of degradation, resulting in an index called the General Damage Factor (FGD). The objective of this study is to make a connection between FGD and solar incidence for ceramic cladding facade of a building located in Brasília-DF. The quantification of the agents was performed by hygrothermal simulation, applying the software WUFI. The influence of radiation was determined by daily calculation of the thermal amplitude or Delta T. The Temperature Incidence Index (Iit) was determined, with 4 Delta-T ranges being defined and their occurrence frequencies determined. The final answer is a Dose-Response curve, which exhibits exponential bias and assists in investigating degradation and shelf life.

Keywords: facades, degradation, hygrothermal simulation, dose response. 


\section{INTRODUÇÃO}

Diante dos diversos sistemas que constituem uma edificação, o envelope ou fachada é considerado um dos elementos mais sensíveis ao processo de degradação, visto ser a primeira barreira de proteção da edificação frente a ação de agentes degradantes que podem ser de natureza mecânica, eletromagnéticos, térmicos, químicos ou biológicos (ISO 15868-2, 2012). A exposição a estes agentes pode ser de forma externa ou interna à edificação.

Caracteriza-se, assim, a degradação das fachadas como um processo complexo, desde que ocorre a partir de ações de vários agentes advindos de diferentes naturezas e que ocasionam alterações prejudiciais as propriedades de um determinado componente ou material. (ASTM E632, 1996). Estes agentes podem estar combinados ou não, a sinergia que ocorre no processo pode se destacar e refletir nos níveis de degradação (NASCIMENTO, 2016).

Diversos tipos de revestimento são utilizados como forma de acabamento nas fachadas, sendo os sistemas de revestimento cerâmico um dos mais empregados devido as vantagens que apresentam, relacionadas a manutenibilidade, resistência química e maior durabilidade, em contrapartida apresentam elevada sensibilidade relacionada a sua forma de execução e heterogeneidade dos materiais empregados, que apresentam características físicas e químicas distintas. A interação entre os agentes de degradação e tais sistemas de revestimentos, podem ocasionar o surgimento de anomalias (SOUZA, 2016).

Assim, o conhecimento dos agentes de degradação dos materiais permite uma tomada de decisões na etapa de projeto que oferece maior proteção ao componente, aumentando a sua vida útil nas mesmas condições de exposição (JERNBERG et al., 2004). Nesta linha de investigação, tem se desenvolvido ferramentas que permintem a quantificação e análise da incidência destes agentes e sua respectiva interação com as fachadas das edificações. O software WUFI, desenvolvido pelo Fraunhofer Institute for Building Physics (IBP) na Alemanha permite a avaliação do comportamento higrotérmico de sistemas construtivos, de acordo com a incidência de agentes climáticos como radiação e amplitude térmica, auxiliando na investigação do processo de degradação.

O estudo dos níveis de degradação, resultantes da interação entre o sistema de revestimento e os agentes, tem se tornado possível apartir do desenvolvimento de técnicas que permitem esta investigação. Nesta pesquisa se utilizou a Metodologia de Mensuração da Degradação (MMD), desenvolvida por pesquisadores da Universidade de Brasília (UnB) e descrita em trabalhos como Souza (2016) e Silva (2014), esta metodologia de análise e diagnóstico de degradação vem sendo aperfeiçoada e empregada por pesquisas desenvolvidas no Programa de Pós-Graduação em Estruturas e Construção Civil $(\mathrm{UnB})$.

A metodologia do MMD permite a obtenção de um índice de degradação, o Fator Geral de Degradação (FGD) que é determinado apartir da relação entre a área degradada e a área total da amostra. Dentre as anomalias identificadas e consideradas por esta técnica estão descolamento, fissuras, eflorescência e falhas de rejunte, sendo determinados os níveis de condição e constantes de ponderação de importância para cada uma das anomalias mensionadas (SOUZA, 2016).

A partir deste índice é possível traçar uma curva de degradação característica para o edifício ou amostra de edifícios em estudo, relacionando o FGD com o tempo, tornando possível a análise e previsão da vida útil. De acordo com Gaspar e de Brito (2008) geralmente é possível avaliar o nível de degradação geral de um elemento do edifício a partir de um índice quantitativo que represente o seu desempenho global.

Com isso, o objetivo deste trabalho é realizar o estudo da degradação de um edifício com fachadas em sistema de revestimento cerâmico, localizado em Brasília-DF. Sendo utilizadas as ferramentas de simulação higrotérmica WUFI para quantificação dos agentes de degradação e metodologia MMD, para obtenção do FGD. Ao final serão elaboradas as curvas dose-resposta, de degradação e análise dos fenômenos.

\section{METODOLOGIA}

O procedimento adotado para o estudo da degradação no sistema de fachada em revestimento cerâmico é a simulação computacional, utilizando o programa WUFI ${ }^{\circledR}$ PRO 5.3 (Wärme-Und Feuchtetransport Instationär). Este programa realiza a simulação do comportamento higrotérmico da envoltória da edificação e atende a todos os requisitos e critérios estabelecidos pela norma DIN EN 15026 (2007), associando estes resultados da simulação higrotérmica à degradação dos edifícios na etapa de pós-processamento, sendo para isto aplicada a metodologia MMD para determinação do FGD e previsão da vida útil do sistema. 


\subsection{Caracterização do edifício}

O edifício em estudo está localizado em Brasília - DF, no Setor de Habitações Coletivas Econômicas Sul - Cruzeiro Novo (SHCES) Qd. 1311, Bloco G, possuindo a idade de 18 anos. Este prédio constitui-se de 4 pavimentos e pilotis, assim, a altura representativa do edifício está entre 10 e $20 \mathrm{~m}$, sendo este intervalo adotado na simulação. A figura 1 apresenta a projeção da edificação obtida através de imagens retiradas no Google Maps, atribuindo sua orientação cardeal, com base na metodologia dos quadrantes (SILVA, 2014), as quais são importantes para a ocorrência dos agentes de radiação e de chuva dirigida. Em seguida, a figura 2 apresenta a definição das orientações das fachadas que foram definidas de acordo com a localização do edifício e a definição dos quadrantes.

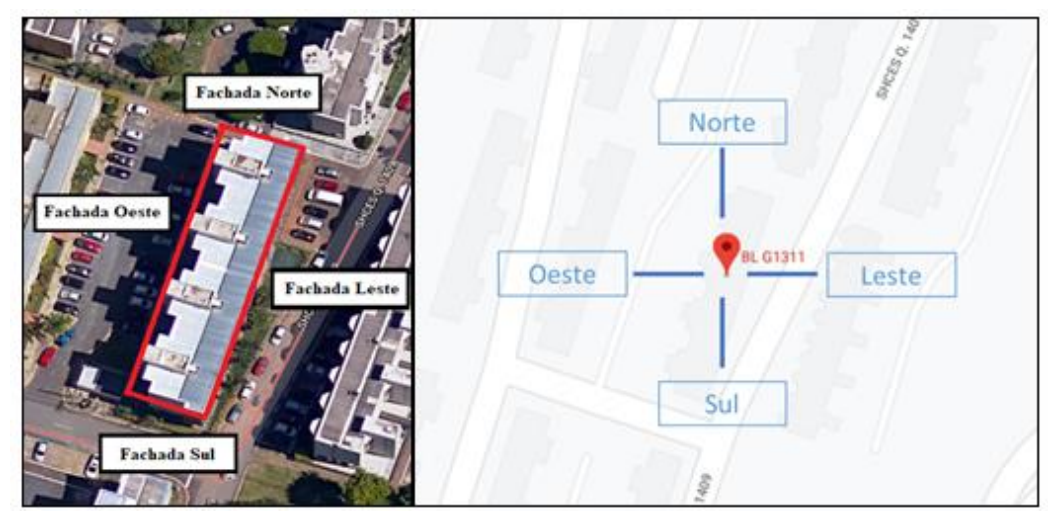

Figura 1: Definição das orientações do edifício (Google Maps, 2019).
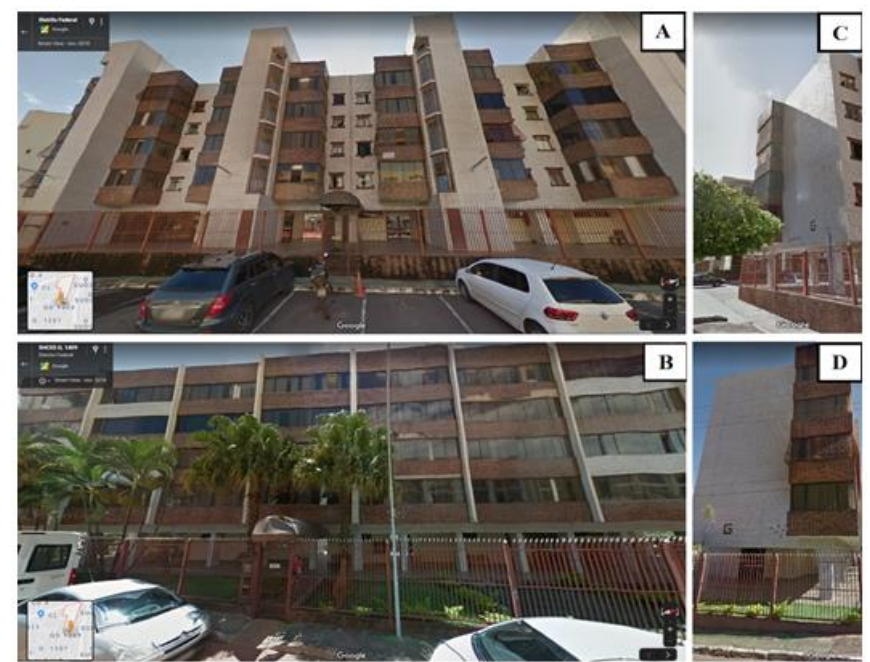

Figura 2: (A) Fachada Oeste; (B) Fachada Leste; (C) Fachada Norte; (D) Fachada Sul (Google Maps, 2019).

Observa-se, na figura 2, que o sistema de vedação é composto por alvenaria de bloco cerâmico revestida externamente (fachadas) por emboço de argamassa mista, com cerâmicas, em dois tons de coloração, telha e branco gelo, com dimensões 10x10 centímetros (nominal), de marca não identificada, assentadas com argamassa colante sobre o emboço. Tendo em vista que não há predominância evidente entre as cores apresentadas nas fachadas, são simuladas as duas cores para as quatros fachadas, totalizando oito casos de estudos.

Para cadastramento dos dados de entrada no software WUFI e definição do sistema construtivo das fachadas do caso em estudo, são estudados dois sistemas: Sistema 01 e Sistema 02. O primeiro é formado por revestimento cerâmico de tom vermelho escuro (cor telha) e o segundo, em tom branco (cor branco gelo), com o intuito de comparar o comportamento higrotérmico em ambos, provocados pela diferença de absortância de cada uma das cores. 


\subsection{Simulação higrotérmica}

A fase de processamento no software WUFI pode ser dividida em três etapas conforme a figura 3. A primeira etapa consiste nos dados de entrada, onde é realizada a caracterização do sistema e das propriedades dos materiais constituintes de cada camada, assim como determinação de coeficientes e do clima onde o edifício em estudo está localizado. A segunda etapa se refere ao procedimento da simulação e obtenção dos dados de saída, que neste estudo se limitam aos dados de radiação, temperatura superficial e amplitude térmica. A terceira e última etapa está relacionada ao pós-processamento, onde ocorre o tratamento dos dados de saída e correlação com os possíveis mecanismos de degradação.

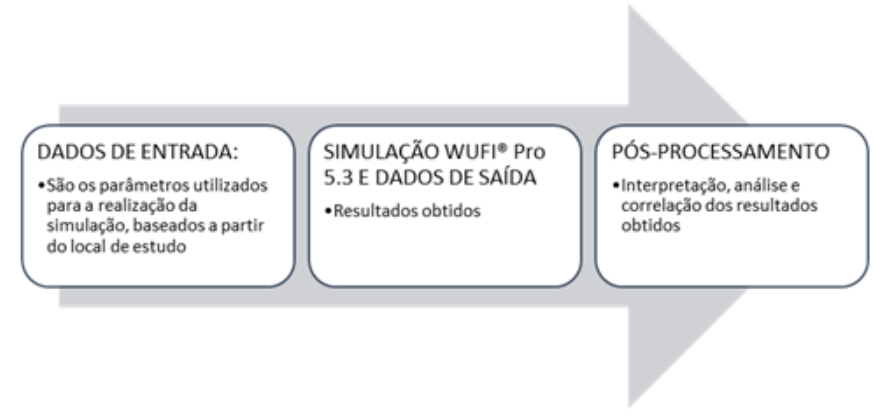

Figura 3: Etapas da simulação (Os autores).

\subsubsection{Caracterização do sistema de revestimento simulado}

O sistema adotado consiste em vedação vertical com acabamento exterior em revestimento cerâmico e interior em revestimento argamassado. Na localidade de Brasília-DF, esta tipologia de sistema é uma das mais usuais para fachadas. A figura 4 apresenta uma representação da seção transversal dos sistemas e o posicionamento de suas câmeras, onde são definidos os dados de entrada com informações dos elementos construtivos e camadas, bem como dados referentes às propriedades higrotérmicas de seus materiais, conforme a tabela 1.

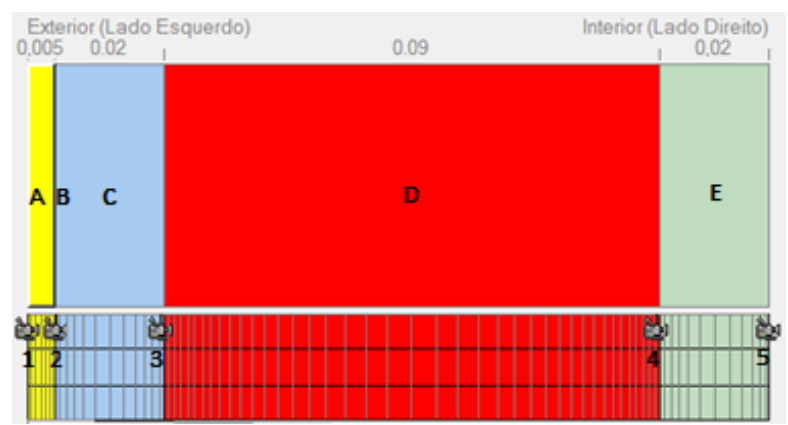

Figura 4: Representação da seção transversal dos sistemas e posicionamento das câmeras de monitorização (adaptado de WUFI, 2013).

O sistema em revestimento cerâmico espessura aproximada de $14 \mathrm{~cm}$ - Sistema 01 e 02:

- Placa cerâmica - 0,5 cm (camada A);

- Argamassa colante - 0,2 cm (camada B);

- Argamassa mista externa - 2,0 cm (camada C);

- Bloco cerâmico - 9,0 cm (camada D);

- Argamassa mista interna - 2,0 cm (camada E).

Além disso, são definidas a utilização de 5 posições de monitoramento nas camadas dos sistemas (superfície exterior, nas interfaces e superfície interior), entretanto, para efeitos de análise são considerados apenas os resultados de temperatura referentes a posição 1 ou superfície exterior.

$\mathrm{Na}$ definição das propriedades higrotérmicas dos materiais, são utilizados dados de estudos anteriores (Tabela 1), realizados no Laboratório de Ensaio de Materiais da Universidade de Brasília (LEM-UnB), para caracterização das propriedades utilizadas na simulação. 
Tabela 1- Propriedades higrotérmicas dos materiais (LEM-UnB).

\begin{tabular}{c|c|c|c|c|c|c|c|c}
\hline & $\begin{array}{c}\text { Massa } \\
\text { específica } \\
\text { aparente }\end{array}$ & Porosidade & $\begin{array}{c}\text { Calor } \\
\text { específico }\end{array}$ & $\begin{array}{c}\text { Condutivi } \\
\text { dade } \\
\text { térmica }\end{array}$ & $\begin{array}{c}\text { Fator de } \\
\text { resistência } \\
\text { à difusão } \\
\text { de vapor } \\
\text { de água }\end{array}$ & $\begin{array}{c}\text { Teor de } \\
\text { umidade } \\
\text { de } \\
\text { referência }\end{array}$ & $\begin{array}{c}\text { Umidade } \\
\text { de } \\
\text { saturação } \\
\text { livre }\end{array}$ & $\begin{array}{c}\text { Coeficiente } \\
\text { de } \\
\text { absorção } \\
\text { de água }\end{array}$ \\
\hline $\begin{array}{c}\text { Unidade } \\
\text { Placa } \\
\text { cerâmica }\end{array}$ & $\mathrm{Kg} / \mathrm{m}^{3}$ & $\mathrm{~m}^{3} / \mathrm{m}^{3}$ & $\mathrm{~J} / \mathrm{kg} \cdot \mathrm{K}$ & $\mathrm{W} / \mathrm{m} \cdot \mathrm{K}$ & {$[-]$} & $\mathrm{Kg} / \mathrm{m}^{3}$ & $\mathrm{Kg} / \mathrm{m}^{3}$ & $\mathrm{Kg} / \mathrm{m}^{3} \mathrm{~S}^{\wedge} 0,5$ \\
\hline $\begin{array}{c}\text { Argamassa } \\
\text { externa }\end{array}$ & 1.730 & 0,290 & 868 & 0,492 & 116 & 1,44 & 192,00 & 0,00100 \\
\hline $\begin{array}{c}\text { Bloco } \\
\text { cerâmico }\end{array}$ & 1.830 & 0,275 & 850 & 0,800 & 17 & 15,69 & 263,39 & 0,16260 \\
\hline $\begin{array}{c}\text { Argamassa } \\
\text { interna }\end{array}$ & 578 & 0,486 & 850 & 0,158 & 23 & 13,00 & 193,00 & 0,09000 \\
\hline
\end{tabular}

\subsubsection{Definição dos coeficientes e do clima}

Em relação à transferência de superfície, são utilizados como parâmetros os dados de entrada da resistência superficial externa $\left(R_{\mathrm{se}}\right)$ e interna $\left(\mathrm{R}_{\mathrm{si}}\right)$, absortância $(\alpha)$ e refletividade. Os valores padrões do software adotados para $\mathrm{R}_{\mathrm{se}} \mathrm{e} \mathrm{R}_{\mathrm{si}}$ são de $0,0588 \mathrm{~m}^{2} \mathrm{~K} / \mathrm{W}$ e $0,1250 \mathrm{~m}^{2} \mathrm{~K} / \mathrm{W}$, respectivamente.

Para a definição do valor de absortância correspondente às cores predominantes na fachada, se obtem os valores a partir do trabalho de Dornelles e Roriz (2007) que define 0,375 para tonalidade branco gelo e 0,635 para telha. Já para a refletividade do solo, adotou-se o valor padrão do software de 0,2 que representa a quantidade de radiação proveniente da atmosfera refletida para o edifício.

Nesta etapa, são definidos os dados de entrada para o clima exterior e interior. Dessa forma, utiliza-se o arquivo do ano meteorológico tipo (Typical Meteorological Year - TMY), obtido do Laboratório de Eficiência Energética em Edificações - LABEE, o qual é composto por dados climáticos horários para um ano, totalizando 8.760 dados (ZANONI, 2015).

\subsubsection{Período de simulação}

O período de simulação adotado vai de 01 de abril de 2019 à 30 de março de 2020, correspondendo a um ano e totalizando 8.760 dados horários. Também são definidos os dados de entrada para a umidade inicial e a condição inicial de temperatura, adotando assim, $80 \%$ de umidade relativa (UR) e $20^{\circ} \mathrm{C}$ para a condição inicial de temperatura. Vale ressaltar que esses valores adotados são considerados como representativos para a cidade de estudo (Brasília) e estão conforme especificados pela ANSI/ASHRAE (2009). Por fim, são realizadas simulações para cada orientação cardeal de fachada, sendo Norte, Sul, Leste e Oeste respectivamente. Realiza-se este procedimento para cada absortância adotada, sendo realizadas oito simulações ao final.

\subsection{4 Índice de incidência de temperatura}

Por meio dos dados de saída obtidos pela simulação higrotérmica, já na etapa de pós-processamento com o objetivo de caracterizar à incidência do clima sobre os edifícios, utiliza-se o índice de incidência de temperatura $\left(\mathrm{I}_{\mathrm{it}}\right)$ que leva em consideração os efeitos dos ciclos (frequência) e valores alcançados de temperaturas nos sistemas. Segundo Haagenrud (2004), pode se utilizar a temperatura para expressar a severidade dos agentes climáticos e para a elaboração deste índice são utilizados os resultados de variação de temperatura $(\Delta \mathrm{T})$. A escolha deste parâmetro é baseada na capacidade de representar as mudanças devido aos ciclos de variação climática. Desse modo, para a elaboração do índice, Nascimento (2016) divide a faixa de variação de temperatura em quatro intervalos iguais, da seguinte maneira:

- Faixa 1: $3,4^{\circ}-11,5^{\circ} \mathrm{C}$;

- Faixa $2: 11,5^{\circ}-19,6^{\circ} \mathrm{C}$;
- Faixa $3: 19,6^{\circ}-27,7^{\circ} \mathrm{C}$;

- Faixa $4: 27,7^{\circ}-35,8^{\circ} \mathrm{C}$

Diante disso, a análise do Iit consiste na mesma metodologia proposta por Nascimento (2016). Portanto, são utilizadas as faixas de variação de temperatura apresentadas acima e o índice calculado de acordo com a equação 1. 


$$
I_{i t}=\sum \frac{\Delta T_{\text {médio.fn }}}{f_{\text {total }}}
$$

Onde:

$\mathrm{I}_{\mathrm{it}}$ é o índice de incidência de temperatura $\left({ }^{\circ} \mathrm{C}\right)$;

$\Delta \mathrm{T}_{\text {médio }}$ é a amplitude de temperatura média da faixa de ocorrência $\left({ }^{\circ} \mathrm{C}\right)$;

$\mathrm{f}_{\mathrm{n}}$ é a frequência de ocorrência da faixa $\mathrm{n}$ de $\Delta \mathrm{T}$;

$\mathrm{f}_{\text {total }}$ é a frequência de todas as ocorrências das quatro faixas de estudo $\left(\mathrm{f}_{\text {total }}=365\right)$.

Como forma de representar a incidência total do edifício em estudo, adota-se também o índice de incidência de temperatura total ( $\mathrm{I}_{\text {itTotal }}$ ), o qual é determinado multiplicando-se os resultados obtidos de $\mathrm{I}_{\text {it }}$ pela idade do edifício. Para o caso do edifício em estudo, adota-se a idade de 18 anos, assim também como as idades de 10, 20, 30 e 40 anos. Dessa forma, se quantifica inicialmente as ocorrências das faixas de $\Delta \mathrm{T}\left({ }^{\circ} \mathrm{C}\right)$ para obter a composição do $\mathrm{I}_{\mathrm{it}}$, e em seguida determina-se o índice considerando as quatro fachadas e respectivos anos. A determinação deste índice permite gerar uma curva dose-reposta, quando analisado em conjunto com o resultados de FGD.

\subsection{Método de mensuração da degradação (MMD)}

A metodologia do MMD é empregada para estudo do grau de desenvolvimento da degradação do prédio investigado. Esta técnica consiste em cinco etapas que são a investigação documental, investigação de campo, ensaios laboratoriais, mapeamento dos danos e diagnóstico. A etapa de mapeamento dos danos consiste na sobreposição de uma malha de $0,25 \mathrm{~m}^{2}(0,50 \times 0,50 \mathrm{~m})$ que permite a quantificação tanto das áreas degradadas quanto da área total. Este mapeamento é feito por zonas, sendo: paredes contínuas (PC), aberturas (AB), sacadas (SC), cantos e extremidades (CE), transição entre pavimentos (TP) e topo (TO).

A investigação do grau da degradação por zonas da fachada permite a obtenção de um indicador de degradação, denominado Fator Geral de Degradação (FGD) que possibilita uma relação entre a gravidade da área que apresenta degradação e a pior condição de degradação encontrada na fachada, seus respectivos níveis de importância relativa e coeficientes de ponderação (SOUZA, 2016). Para sistemas em revestimento cerâmico as anomalias consideradas são descolamento cerâmico (DC), fissuração (FI), falha de rejunte (FR) e eflorescência (EF), sendo o cálculo deste indicador realizado conforme a equação 2 .

$$
F G D=\frac{A d_{D C} \cdot k n_{D C} \cdot k c n_{D C}+A d_{F I} \cdot k n_{F I} \cdot k c n_{F I}+A d_{F R} \cdot k n_{F R} \cdot k c n_{F R}+A d_{E F} \cdot k n_{E F} \cdot k c n_{E F}}{\sum k_{\text {máx }} \cdot A t}
$$

Onde:

FGD é o Fator Geral de Degradação;

Ad é a área que apresenta anomalia $\left(\mathrm{m}^{2}\right)$;

kn é o nível de condição da anomalia, variando entre 1, 2, 3 e 4;

kcn é a importância relativa da anomalia;

kmáx é a constante de ponderação referente ao nível de pior condição da anomalia $\left(4_{\mathrm{DC}}+4_{\mathrm{FI}}+3_{\mathrm{FR}}+3_{\mathrm{EF}}=14\right)$;

At é a área total da fachada $\left(\mathrm{m}^{2}\right)$.

Sendo apresentados na tabela 2 os valores referentes aos níveis de condição e importância relativa para cada tipologia de anomalia investigada. 
Tabela 2- Valores de ponderação para FGD (SOUZA, 2016).

\begin{tabular}{c|c|c|c}
\hline Defeitos & knc & Nível de condição (kn) & Área afetada $\left.\mathbf{( m}^{\mathbf{2}}\right)$ \\
\hline Descolamento cerâmico & 0,82 & Nível 2 & $<37,14 \%$ \\
& & Nível 3 & $37,14 \%<\mathrm{x}>49,14 \%$ \\
& & Nível 4 & $>49,14 \%$ \\
\hline Fissuração & 1,00 & Nível 2 & $<4 \%$ \\
& & Nível 3 & $4 \%<\mathrm{x}>6,05 \%$ \\
& & Nível 4 & $>6,05 \%$ \\
\hline Falha de rejunte & 0,06 & Nível 1 & $<4,32 \%$ \\
& & Nível 2 & $4,32 \%<\mathrm{x}>9,04 \%$ \\
& & Nível 3 & $>9,04 \%$ \\
\hline Eflorescência & 0,12 & Nível 1 & $<1,18 \%$ \\
& & Nível 2 & $1,18 \%<\mathrm{x}>1,47 \%$ \\
& & Nível 3 & $>1,47 \%$ \\
\hline
\end{tabular}

\section{RESULTADOS E DISCUSSÃO}

A partir da simulação higrotérmica são obtidos os resultados para radiação solar, temperatura superficial e amplitude térmica, considerando os dois valores de absortância. Os resultados permitem avaliar a influência da radiação sobre o sistema de revestimento cerâmico estudado e também a relevância dos diferentes teores de absortância, analisando como essa diferença impacta nas solicitações sobre os revestimentos. A figura 5 apresenta os dados obtidos para radiação global acumulada mensal, composta pelas parcelas de radiação direta, difusa e refletida, para cada orientação de fachada.

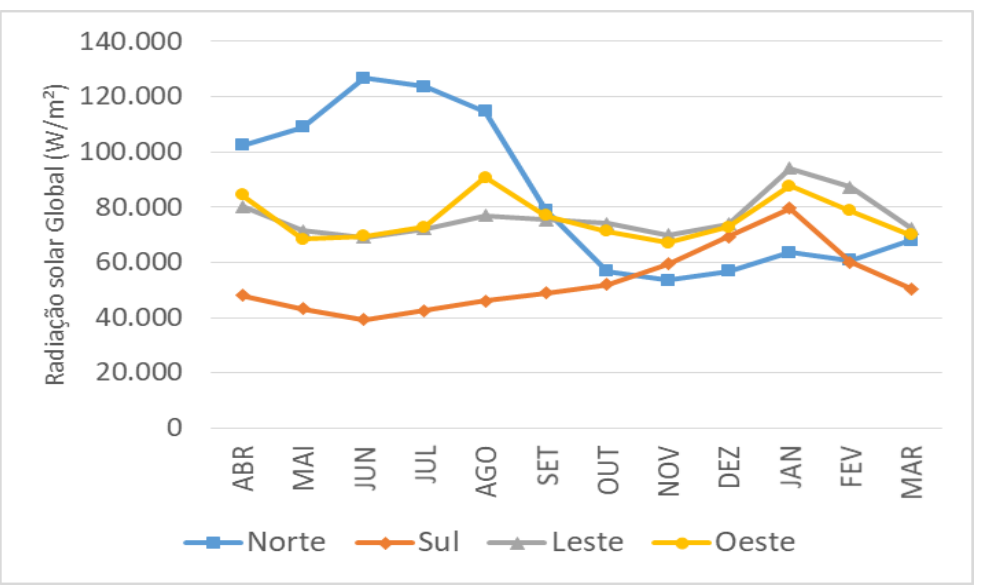

Figura 5: Perfil anual da Radiação Solar Global mensal por orientação (Os autores).

A localidade em estudo Brasília-DF é caracterizada por apresentar um período seco que equivale ao tempo decorrido entre abril e setembro e um tempo chuvoso, que corresponde de outubro a março, sendo este fenômeno comprovado por estudos de Zanoni (2015). A partir da figura 5, nota-se que a fachada Norte foi a que mais recebeu incidência da radiação solar ao longo do período seco do ano, no entanto a incidência de radiação diminui consideravelmente durante o período chuvoso, e passa a ser predominante nas fachadas Leste, Oeste e Sul, respectivamente. Observa-se que as orientações de fachada Leste e Oeste não apresentam variações consideráveis ao longo do ano, mantendo uma tendência de comportamento. A fachada Norte se mostra como a mais influenciada pelo fenômeno da sazonalidade, passando de um período com forte incidência solar, para outro com uma redução considerável desta. Tal influência pode ser responsável pelo surgimento de anomalias, uma vez que esta interação revestimento e agentes de forma cíclica, ocasiona variações térmicas e respectivas movimentações, podendo gerar fenômenos de retração e expansão, causando danos ao sistema de revestimento.

Paralelamente, são analisados também os resultados para temperatura superficial, considerando a máxima temperatura superficial mensal, para cada orientação de fachada, considerando os dois valores de absortância, segundo as figuras 6 (a) (b). 

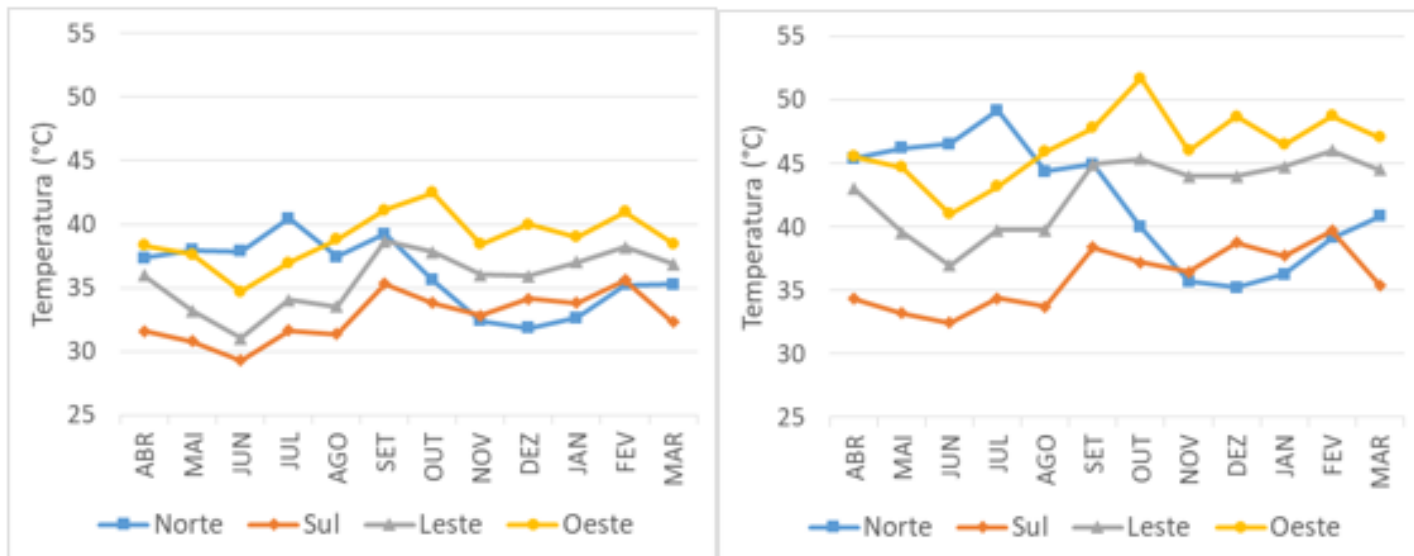

Figura 6: Perfil anual da temperatura superficial máxima mensal para as quatro orientações para o revestimento de cor branco gelo (a) e revestimento de cor telha (b) (Os autores).

A partir da figura 6 é possível observar a influência direta dos valores de absortância sobre os valores de temperatura superficial. Tanto a figura 6 (a) quanto a figura 6 (b) apresentam a orientação de fachada Oeste com os maiores resultados, seguida da Leste, porém para a absortância de cor telha os valores alcançados são bastante superiores quando comparados a absortância em cor branco gelo, sendo assim comprovado que quanto mais próximo do valor de 1 a absortância consegue absorver maior radiação.

Outro fator a ser considerado em relação a fachada Oeste é que esta recebe a incidência da radiação no período vespertino, sendo este caracterizado por apresentar os maiores valores de temperatura ao longo do dia, impactando na temperatura superficial do revestimento. Pode se observar também, assim como na figura 5, que o comportamento da fachada Norte é maior no período seco e diminui no período chuvoso, o que é contrário para a fachada Sul, que apresenta um comportamento crescente entre período seco e chuvoso. As máximas temperaturas superficiais na cor telha chegaram a ser $20 \%$ maior em comparação com a cor branco gelo, sendo que quanto maior a absorção de radiação de um revestimento, maior será sua probabilidade de apresentar anomalias por variações térmicas que ocasionam tensões sobre o revestimento.

Aliado aos resultados de temperatura superficial se encontram os dados de amplitude térmica. O cálculo da amplitude térmica é realizado considerando o gradiente de temperatura formado a partir da diferença entre a máxima e a mínima temperatura superficial diária. Os resultados obtidos são apresentados na figura 7 (a) (b).

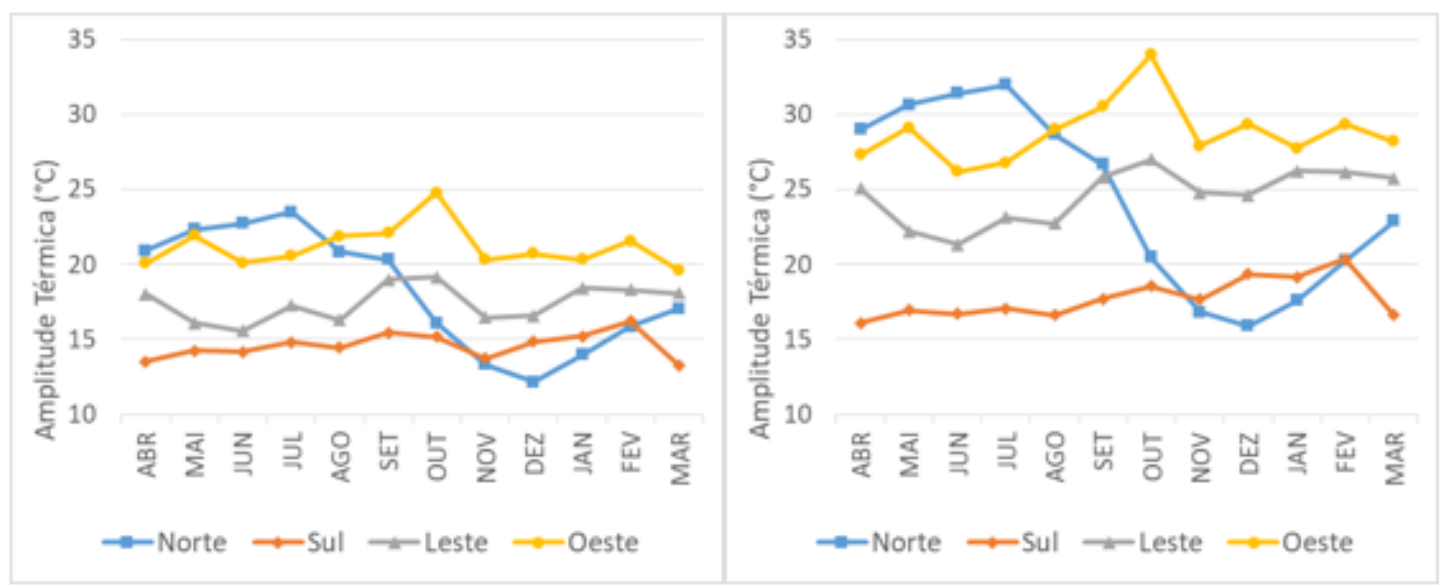

Figura 7: Perfil anual das amplitudes térmicas máximas mensais para as quatro orientações para o revestimento de cor branco gelo (a) e revestimento de cor telha (b) (Os autores).

A influência da incidência de radiação e dos valores de temperatura superficial alcançados são relevantes quando analisados com base no fenômeno de amplitude térmica, uma vez que este mensura a variação de temperatura que ocorre na camada de revestimento. Esta oscilação na temperatura da camada de revestimento de forma diária e cíclica, gera solicitações que podem ser a origem do surgimento de manifestações patológicas. É possível constatar assim como para 
a temperatura superficial, quanto maior o valor do coeficiente de absortância, maior serão os gradientes de temperatura formados e maiores as solicitações, ainda mais considerando que os resultados de amplitude térmica na superfície da fachada de cor telha chegaram a cerca de $37 \%$ maior em comparação com a cor branco gelo. No período seco a fachada que apresenta os maiores valores de amplitude é a Norte, seguida da Oeste, Leste e Sul, respectivamente. Porém, no período chuvoso esta configuração é alterada, sendo composta por fachada Oeste, Leste, Norte e Sul, respectivamente.

Com base nos dados mensurados pela simulação higrotérmica, são obtidos os índices de incidência de temperatura ( $\left.\mathrm{I}_{\mathrm{it}}\right)$ para ambos coeficientes de absortância, cor branco gelo e telha, apresentados na tabela 3. Estes resultados são analisados em conjunto com os resultados de FGD, apresentados na tabela 4, e posteriormente analisados para investigar o grau de degradação das orientações de fachada.

Tabela 3- Valores de Iit para absortâncias distintas (Os autores).

\begin{tabular}{c|c|c|c|c|c|c|c|c}
\hline \multirow{2}{*}{$\begin{array}{c}\text { IDADE } \\
\text { (ANOS) }\end{array}$} & \multicolumn{3}{|c|}{ COR BRANCO GELO } & \multicolumn{4}{c}{ COR TELHA } \\
\cline { 2 - 9 } Iit & NORTE & SUL & LESTE & OESTE & NORTE & SUL & LESTE & OESTE \\
\hline $\mathbf{1 0}$ & 26,93 & 12,13 & 13,82 & 15,26 & 20,17 & 14,91 & 19,66 & 21,19 \\
\hline $\mathbf{1 8}$ & 149,30 & 218,42 & 248,77 & 274,74 & 363,02 & 268,35 & 353,83 & 381,39 \\
\hline $\mathbf{2 0}$ & 298,61 & 242,34 & 138,21 & 152,63 & 201,68 & 149,08 & 196,57 & 211,88 \\
\hline $\mathbf{3 0}$ & 447,91 & 276,42 & 305,27 & 403,35 & 298,16 & 393,15 & 423,77 \\
\hline $\mathbf{4 0}$ & 597,22 & 484,03 & 414,62 & 457,90 & 605,03 & 447,25 & 589,72 & 635,65 \\
\hline
\end{tabular}

Tabela 4- Valores de FGD para orientações de fachada (Os autores).

\begin{tabular}{c|c|c|c|c}
\hline Anos & NORTE & SUL & LESTE & OESTE \\
\hline $\mathbf{1 0}$ & 0,003 & 0,001 & 0,008 & 0,001 \\
\hline $\mathbf{1 8}$ & 0,007 & 0,002 & 0,019 & 0,002 \\
\hline $\mathbf{2 0}$ & 0,008 & 0,002 & 0,022 & 0,002 \\
\hline $\mathbf{3 0}$ & 0,016 & 0,004 & 0,041 & 0,005 \\
\hline $\mathbf{4 0}$ & 0,027 & 0,007 & 0,066 & 0,008 \\
\hline
\end{tabular}

Os resultados de $\mathrm{I}_{\mathrm{it}}$ e $\mathrm{I}_{\mathrm{itTOTAL}}$ evidenciam a sinergia entre a radiação e intensidade de incidência das temperaturas. As orientações Norte, Oeste e Leste apresentam crescimento gradual e considerável ao longo dos anos e quando os resultados são avaliados considerando as diferentes absortâncias, é evidente a influência do maior valor de absortância no aumento do índice Iit. Revestimentos cerâmicos de fachada em cores escuras, de acordo com os dados obtidos, estão mais propícios a influência da incidência de temperatura e possível aumento no surgimento de anomalias como descolamento e fissuras.

A análise do FGD de acordo com a tabela 4 também apresenta dados com avanço gradual, o que é esperado uma vez que os dados obtidos partem de uma equação previamente estabelecida e elaborada para a realidade da localidade de BrasíliaDF. A fachada Leste apresenta os maiores índices de degradação, para as diferentes idades, seguida da fachada Norte, Oeste e Sul, respectivamente.

A elaboração da curva dose-resposta para o edifício em estudo aponta o comportamento da degradação das fachadas quando considerado o índice de degradação de íncidência de temperatura. Para a idade do edífício de 18 anos, o valor do Iit desempenho do revestimento. O valor de FGD para esta idade é igual a 0,019. Quando comparada a orientação de fachada Norte, sendo esta a segunda mais influenciada pelo índice, os valores do Iit TотAL para 18 anos são 149,30 para cor branco gelo e 201,68 para cor telha, já o FGD apresentou o valor de 0,007. A figura 8 apresenta a configuração obtida para a

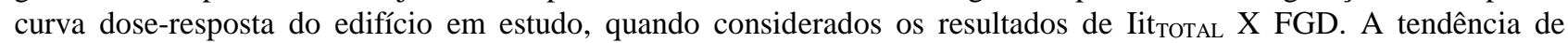
degradação observada é crescente, o que já é esperado uma vez que as edificações tendem a apresentar perca de desempenho quanto maior a ação de agentes degradantes. 


\section{СВРAT 2020 \\ CONGRESSO BRASILEIRO DE PATOLOGIA DAS CONSTRUÇÕES

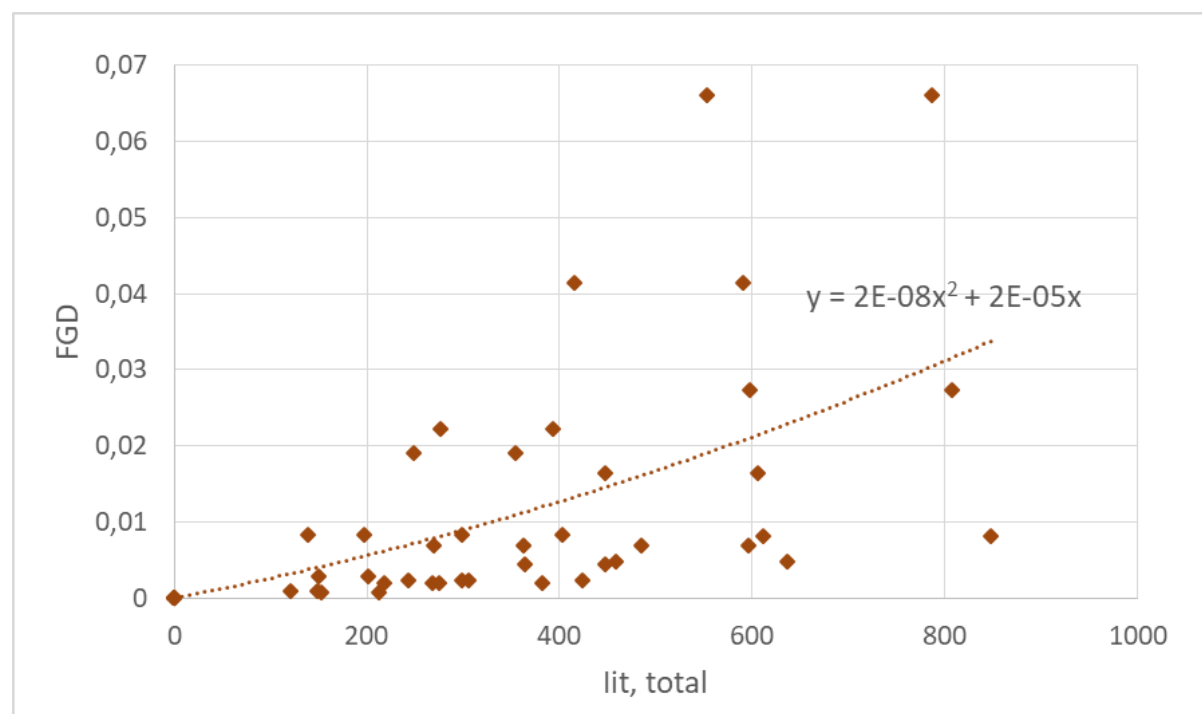

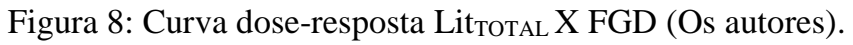

Outra abordagem do FGD consiste na avaliação da vida útil, por ser um índice obtido apartir da técnica de inspeções de edifícios (SOUZA, 2016). Neste estudo foi adotada a referência normativa da ABNT NBR 15575:1 (2013), de forma semelhante a adotada por Silva (2014), onde para revestimentos de fachadas aderentes é determinada uma vida útil de projeto mínima de 20 anos. Assim, a figura 9 apresenta uma curva de degradação geral do edifício, considerando todos os dados de FGD para cada orientação e idades de 10, 18 (idade do edifício), 20, 30 e 40 anos.

A partir desta curva foi identificado o valor correspondente ao FGD para a idade de 20 anos, sendo 0,0088. Este valor correponde ao limite de degradação aceitável. Com base nisto é possível concluir, tendo como referência a tabela 4 que na idade de 18 anos, correspondente a idade atual do edifício a orientação de fachada Leste já se encontra ultrapassando o limite de degradação aceito, com o valor de 0,019. A orientação Norte irá atingir este limite a partir do momento que o edifício atingir a idade de 30 anos, com o valor de 0,016 e as demais orientações Oeste e Sul, ao longo dos 40 anos investigados, não irão atingir esta demarcação. Sendo assim, aqueles que ultrapassaram este limite, possivelmente extrapolaram (de forma negativa) o limite mínimo de vida útil de projeto, eventualmente havendo a necessidade manutenção ou em casos extremos de degradação, a substituição do sistema de revestimento cerâmico (SOUZA, 2016).

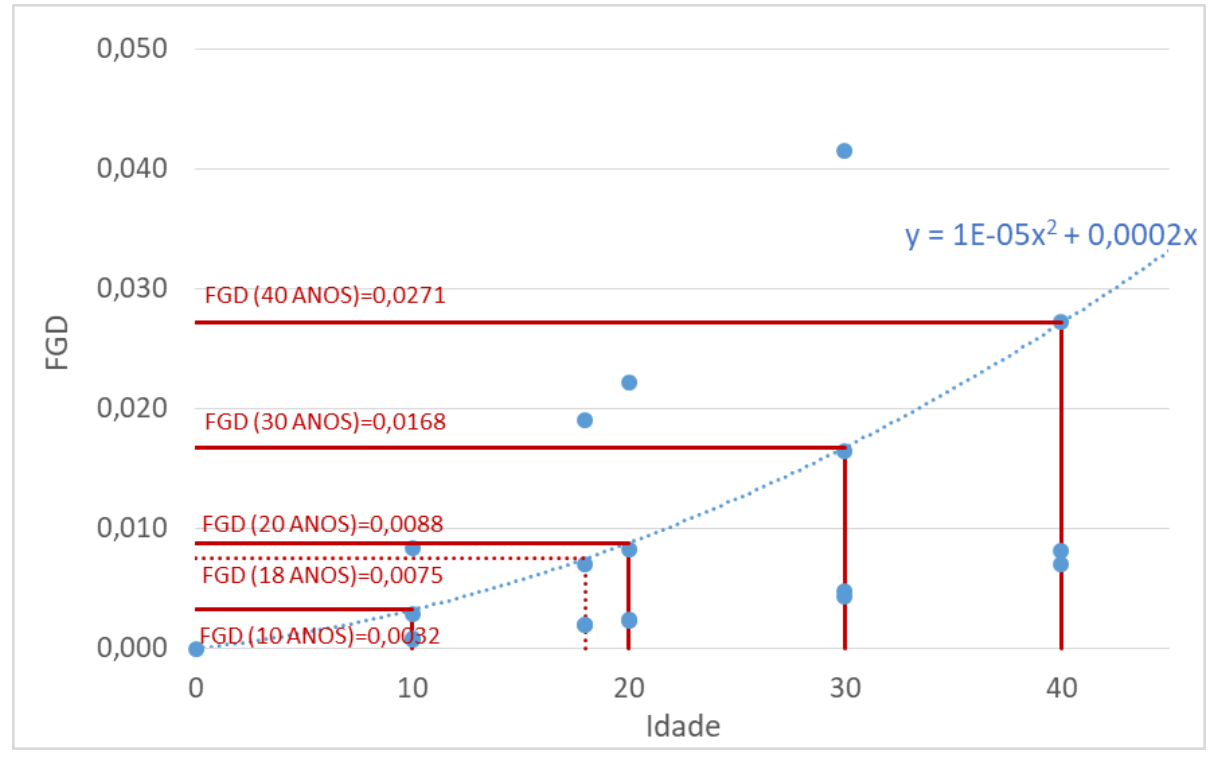

Figura 9: Dados de FGD X tempo - previsão de vida útil (Os autores). 


\section{CONCLUSÕES}

Em vista disso, a presente investigação permitiu enumerar as seguintes conclusões:

- A orientação de fachada Norte foi a que mais recebeu incidência da radiação solar ao longo do período seco do ano, porém no período chuvoso passa a ser predominante nas fachadas Leste, Oeste e Sul, respectivamente;

- Em termos de temperatura superficial e amplitude térmica, constatou-se a influência direta dos coeficientes de absortância sobre os valores obtidos. Quanto maior o coeficiente, maior a capacidade de absorção de radiação do revestimento;

- Os índices Iit para as diferentes idades, evidenciaram que os revestimentos em cor telha (maior coeficiente de absortância), apresentam maior probabilidade do surgimento de anomalias ocasionadas por movimentações térmicas, gerando tensões;

- A curva dose-resposta apontou uma tendência de degradação crescente, quando considerados os resultados do IitToTAL em conjunto com o FGD;

- A previsão de vida útil a partir dos resultados de FGD X Idade, demostrou que a fachada Leste já se encontra ultrapassando o limite de degradação aceitável e que a fachada Norte irá atingir este limite na idade de 30 anos;

- A curva dose-resposta e análise do FGD X Idade, consistem em metodologias efetivas para avaliação do grau de degradação e nível de desempenho das edificações.

\section{AGRADECIMENTOS}

O presente trabalho foi realizado com apoio da Coordenação de Aperfeiçoamento de Pessoal de Nível Superior - Brasil (CAPES) - Código de Financiamento 001.

\section{REFERÊNCIAS}

AMERICAN SOCIETY FOR TESTING AND MATERIALS. ASTM - E 632-82: Standard Practice for Developing Accelerated Tests to aid Prediction of the Service Life of Building Components and Materials. Philadelphia, 1996.

ASSOCIAÇÃO BRASILEIRA DE NORMAS TÉCNICAS - ABNT NBR 15575-1: Edificações Habitacionais Desempenho. Requisitos Gerais. Rio de Janeiro, 2013.

ANTUNES, G. R. Estudo de manifestações patológicas em revestimento de fachada em Brasília: sistematização da incidência de casos. Dissertação (Mestrado). Universidade de Brasília, 2010.

DEUTSCHES INSTITUT FÜR NORMUNG. DIN EN 15026 Hygrothermal performance of building components and building elements - Assessment of moisture transfer by numerical simulation. CEN, Brussels, Belgium, 2007.

DORNELLES, K. A.; RORIZ, M. Influência das tintas imobiliárias sobre o desempenho térmico e energético de edificações. X Congresso Internacional de Tintas. Anais...São Paulo. 2007.

GASPAR, P. L.; BRITO, J. Quantifying environmental effects on cement-rendered facades: A comparison between different degradation indicators. Building and Environment, v. 43, p. 1818-1828, 2008.

HAAGEnRUD, S. Factors Causing Degradation: Part II. In: Guide and Bibliography to Service Life and Durability Research for Buildings and Components. CIB Publication 295, p.1.2-104. CIB, Rotterdam, March 2004.

ISO/DIS 15686-2: Buildings - Service life planning. Part 2: Service life prediction procedures, International Organization for Standardization, 2012. 
JERNBERG, P.; SJÖSTRÖM, C.; LACASSE, M. A.; BRANDT, E.; SIEMES, T. Service life and durability research. In: Guide and Bibliography to Service Life and Durability Research for Buildings and Components. CIB Publication 295, p.11-59. CIB, 2004.

NASCIMENTO, M. L. M. (2016). Aplicação da Simulação Higrotérmica na Investigação da Degradação de Fachadas de Edifícios. Dissertação de Mestrado em Estruturas e Construção Civil, Publicação E.DM-018A/16, Departamento de Engenharia Civil e Ambiental, Universidade de Brasília, Brasília, DF, 173p.

PINHEIRO, Pedro Ivo Santos (2016). Aplicação do Método de Mensuração da Degradação (MMD) ao Estudo das Fachadas de Edifícios em Brasília. Monografia de Projeto Final, Publicação, Departamento de Engenharia Civil e Ambiental, Universidade de Brasília, Brasília, DF, 83p.

SILVA, M. N. B. Avaliação quantitativa da degradação e vida útil de revestimentos de fachada - aplicação ao caso de Brasília/DF. Tese (Doutorado) Universidade de Brasilia, 2014.

SOUZA, J.S. Evolução da degradação de fachadas - efeito dos agentes de degradação e dos elementos constituintes. Dissertação de Mestrado em Estruturas e Construção Civil, Departamento de Engenharia Civil e Ambiental, Universidade de Brasília, Brasília, DF, 2016. 114 p.

WUFI Pro 6.1. IBP - Fraunhofer Institute for Building Physics. Holzkirchen, Germany, 2013.

ZANONI, V. A. G. Influência dos agentes climáticos de degradação no comportamento higrotérmico de fachadas em Brasília. Tese (Doutorado) Universidade de Brasília, 2015. 